\title{
Alloy fluctuations at dislocations in III- nitrides: identification and impact on optical properties
}

F. C.-P. Massabuau, P. Chen, S. L. Rhode, M. K. Horton,

T. J. O'Hanlon, et al.

F. C.-P. Massabuau, P. Chen, S. L. Rhode, M. K. Horton, T. J. O'Hanlon, A. Kovács, M. S. Zielinski, M. J. Kappers, R. E. Dunin-Borkowski, C. J. Humphreys, R. A. Oliver, "Alloy fluctuations at dislocations in III-nitrides: identification and impact on optical properties," Proc. SPIE 10532, Gallium Nitride Materials and Devices XIII, 105320R (23 February 2018); doi: 10.1117/12.2288211

SPIE. Event: SPIE OPTO, 2018, San Francisco, California, United States 


\title{
Alloy fluctuations at dislocations in III-Nitrides: identification and impact on optical properties
}

\author{
F.C-P. Massabuau ${ }^{\mathrm{a}}$, P. Chen ${ }^{\mathrm{a}}$, S.L. Rhode ${ }^{\mathrm{b}}$, M.K. Horton ${ }^{\mathrm{c}}$, T.J. O'Hanlon ${ }^{\mathrm{a}}$, A. Kovács ${ }^{\mathrm{d}}$, M.S. \\ Zielinski $^{\mathrm{e}}$, M.J. Kappers ${ }^{\mathrm{a}}$, R.E. Dunin-Borkowski ${ }^{\mathrm{d}}$, C.J. Humphreys ${ }^{\mathrm{a}}$, and R.A. Oliver ${ }^{\mathrm{a}}$ \\ aDepartment of Materials Science and Metallurgy, University of Cambridge, Cambridge CB3 \\ OFS, UK \\ ${ }^{\mathrm{b}}$ Department of Materials, Imperial College London, London SW7 2AZ, UK \\ ${ }^{c}$ Materials Science and Engineering, University of California Berkeley, Berkeley, CA 94720, US \\ ${ }^{\mathrm{d}}$ Ernst Ruska-Centre for Microscopy and Spectroscopy with Electrons and Peter Grünberg \\ Institute, Forschungszentrum Jülich GmbH, D-52425 Jülich, Germany \\ eAttolight AG, EPFL Innovation Park, 1015 Lausanne, Switzerland
}

\begin{abstract}
We investigated alloy fluctuations at dislocations in III-Nitride alloys (InGaN and AlGaN). We found that in both alloys, atom segregation (In segregation in InGaN and Ga segregation in AlGaN) occurs in the tensile part of dislocations with an edge component. In InGaN, In atom segregation leads to an enhanced formation of In-N chains and atomic condensates which act as carrier localization centers. This feature results in a bright spot at the position of the dislocation in the CL images, suggesting that non-radiative recombination at dislocations is impaired. On the other hand, Ga atom segregation at dislocations in AlGaN does not seem to noticeably affect the intensity recorded by CL at the dislocation. This study sheds light on why InGaN-based devices are more resilient to dislocations than AlGaN-based devices. An interesting approach to hinder non-radiative recombination at dislocations may therefore be to dope AlGaN with In.
\end{abstract}

Keywords: dislocation, III-Nitrides, segregation, carrier localization, aberration-corrected TEM, cathodoluminescence.

\section{INTRODUCTION}

III-Nitrides are a hugely important family of semiconductors which mainly consists of GaN and its alloys with InN and AlN. While the former enables the fabrication of efficient blue and green light emitting diodes (LEDs), ${ }^{1}$ the latter holds the key for the fabrication of ultraviolet (UV) LEDs. ${ }^{2}$ It is common knowledge that the efficiency of an LED varies substantially with its emission wavelength $\lambda$, which is controlled for the most part by its alloy content. For example, external quantum efficiencies of $44 \%,{ }^{3} 84 \%^{4}$ and $20 \%{ }^{5}$ have been reported for InGaN green LED $(\lambda \sim 527 \mathrm{~nm})$, InGaN blue LED $(\lambda \sim 440 \mathrm{~nm})$, and AlGaN UV LED $(\lambda \sim 275 \mathrm{~nm})$, respectively. III-Nitride materials are also plagued by a high density of dislocations, classically in the range of $10^{7}-10^{10} \mathrm{~cm}^{-2}$, which act as non-radiative recombination centers, ${ }^{6-9}$ and whilst InGaN-based devices exhibit a remarkable resilience to this defect, ${ }^{10}$ dislocations are thought to play an important role in the relatively low efficiency reported in AlGaN-based devices. ${ }^{11}$

In order to better understand the seemingly different behaviors of dislocations in InGaN and AlGaN devices, in this study we compare the structural (alloy distribution) and optical properties of dislocations in InGaN and AlGaN thick films. We report that alloy segregation occurs at the dislocations in both samples, with the largest atom (In in InGaN film, Ga in AlGaN film) segregating in the tensile part of the dislocation. However while this leads to the enhanced formation of localization centers in InGaN, therefore limiting the impact of dislocations on the optical properties of the material, no such improvement was observed in the case of AlGaN.

Further author information:

F.M.: E-mail: fm350@cam.ac.uk, Telephone: +44 (0)1223 334368 


\section{METHODS}

An InGaN and an AlGaN epilayers were grown by metal organic vapor phase epitaxy in a Thomas Swan $6 \times 2$ inch close-coupled showerhead reactor. Trimethylgallium (TMG), trimethylaluminum (TMA), trimethylindium (TMI), diluted silane in hydrogen $\left(\mathrm{SiH}_{4}\right)$, and ammonia $\left(\mathrm{NH}_{3}\right)$ were used as precursors for the $\mathrm{Ga}, \mathrm{Al}, \mathrm{In}, \mathrm{Si}$, and $\mathrm{N}$ elements, respectively. Hydrogen $\left(\mathrm{H}_{2}\right)$ was used as carrier gas for the growth of GaN, AlN, and AlGaN, while a mixture of $\mathrm{H}_{2}$ and nitrogen $\left(\mathrm{N}_{2}\right)$ was employed for the growth of InGaN. A c-plane sapphire substrate was employed for both samples.

For the AlGaN epilayer, following the growth of a $30 \mathrm{~nm}$ AlN buffer layer at $1050^{\circ} \mathrm{C}$, a $2 \mu \mathrm{m} \mathrm{AlN} \mathrm{layer}$ was grown at $1130^{\circ} \mathrm{C} . \mathrm{A} \mathrm{SiN}_{x}$ interlayer was then deposited to allow for the subsequent growth of faceted GaN islands at $980^{\circ} \mathrm{C}$ for $120 \mathrm{~s}$. Finally the $3 \mu \mathrm{m} \mathrm{Al} \mathrm{Ga}_{1-x} \mathrm{~N}$ epilayer with an $\mathrm{Al}$ content of $x \sim 46 \%$ (as determined by X-ray diffraction (XRD)) was deposited at $1090^{\circ} \mathrm{C}$. From XRD analysis, the AlGaN layer was found to be $89 \%$ relaxed with regards to the strain-free AlN layer. Further details about the growth of the sample can be found in Ref. 12 .

For the InGaN epilayer, a $5 \mu \mathrm{m}$ GaN buffer layer (of which $2 \mu \mathrm{m}$ undoped and $3 \mu \mathrm{m}$ Si-doped to $5 \times 10^{18}$ $\mathrm{cm}^{-3}$ ) was first deposited. Following the growth of an additional $500 \mathrm{~nm}$ of GaN, a $135 \mathrm{~nm} \mathrm{In}_{x} \mathrm{Ga}_{1-x} \mathrm{~N}$ epilayer with an In content of $x \sim 9 \%$ (as determined by XRD) was deposited at $749^{\circ} \mathrm{C}$. From XRD analysis, the InGaN layer was found to be fully strained to the strain-free GaN layer. Further details about the growth of the sample can be found in Ref. 13 .

Alloy fluctuations around dislocations in the AlGaN epilayer were assessed using energy dispersive X-ray spectroscopy (EDS) in an FEI Titan G3 80-200 ChemiSTEM microscope operated at $200 \mathrm{kV}$ with detector collection semiangle of 69 mrad. ${ }^{14}$ The sample was observed in plan-view geometry (i.e. dislocations seen end-on) by high-angle annular dark field scanning transmission electron microscopy (HAADF-STEM). EDS quantification was performed using a standard background subtraction and the Cliff-Lorimer factor method.

To correlate the optical and morphological properties of the materials, we employed a "multi-microscopy" approach whereby the same dislocation is analyzed by atomic force microscopy (AFM) and scanning electron microscopy combined with cathodoluminescence (SEM-CL). ${ }^{15}$ AFM was conducted on a Veeco dimension 3100 microscope in tapping mode, and was here used to determine the dislocation type, obtained from the diameter of the V-pit terminating the dislocation. ${ }^{16}$ AFM was also used to measure the distance to nearest neighbor for each dislocation. SEM-CL was conducted on an FEI XL30 microscope equipped with a Gatan MonoCL4 system operated at $3 \mathrm{kV}$ at room temperature (for the InGaN sample), and an Attolight Rosa 4634 CL microscope operated at $3 \mathrm{kV}$ at liquid He $(10 \mathrm{~K})$ temperature (for the AlGaN sample). (The AlGaN epilayer was analyzed at low temperature because the CL signal at room temperature was too low.) CL was used to measure the CL integrated intensity at the dislocation position compared to that of the surrounding material unaffected by the dislocation (thereafter referred as "intensity ratio"), as well as the shift in CL emission energy between these regions (thereafter referred as "energy shift"). A more detailled description of the experiment is provided in Ref. 13.

\section{RESULTS AND DISCUSSION}

\subsection{AlGaN epilayer}

Figure 1 shows an example of TEM observation of dislocation in AlGaN. The dislocation core configuration can be identified in the HAADF-STEM image - here consisting of a mixed-type dislocation with double 5/6-atom ring core. The atomic resolution allows for further treatment to be performed, such as in this case, geometric phase analysis (GPA). GPA allows to draw a qualitative strain map of the region around the dislocation. With regards to the $\epsilon_{x x}$ strain component (with $x=[11 \overline{2} 0]$ ), we can see in Figure 1(b) that the dislocation is bounded on both sides by a region of tensile strain and a region of compressive strain, as expected by the elastic theory for a dislocation with an edge component. An EDS line scan taken across the dislocation core (as shown shematically in Figure 1(b)) reveals alloy fluctuations near the dislocation. As illustrated in Figure 1(c), the composition of the alloy is not constant across the dislocation. We report that the alloy is Ga-rich at the nm-scale on the side of the dislocation corresponding to the region under tensile strain, and Al-rich on the other side of the dislocation corresponding to the region under compressive strain. This result could be explained from an energetic aspect: 

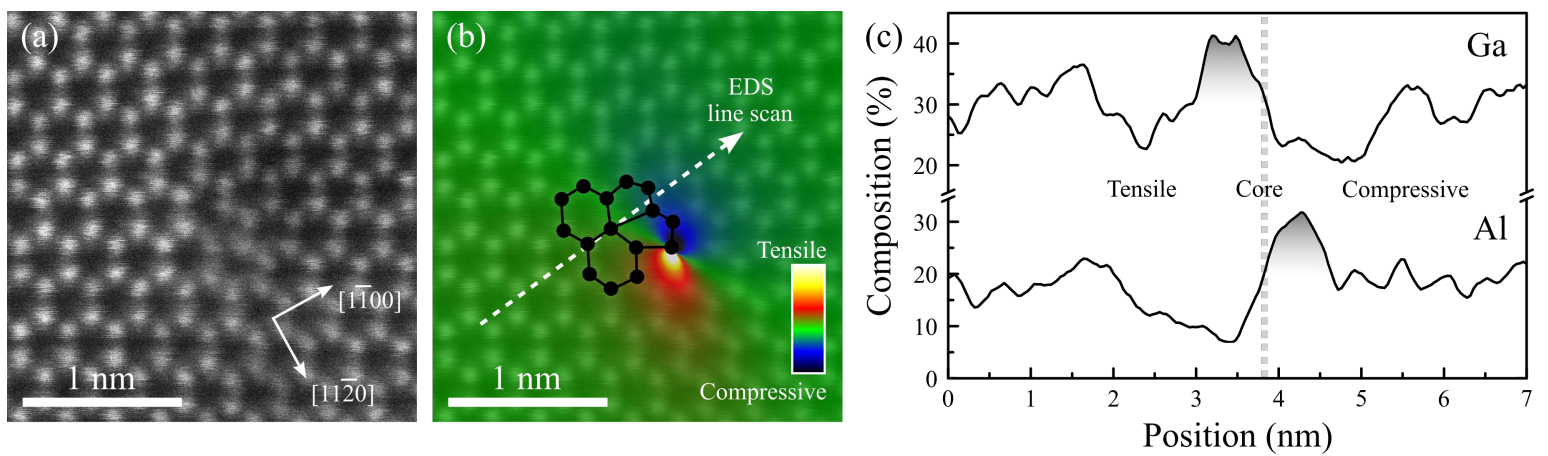

Figure 1. (a) HAADF-STEM image and (b) GPA map of a mixed-type dislocation. The GPA map corresponds to the $\epsilon_{x x}$ strain component, with $x=[11 \overline{2} 0]$. (c) Al and Ga composition profiles obtained from the EDS line scan taken across the dislocation (position shown schematically in (b), with a $1 \mathrm{~nm}$ analysis width).

given that the Ga-N bond is longer than $\mathrm{Al}-\mathrm{N}$ bond, the elastic energy of the dislocation is reduced if the tensile part is Ga-rich and the compressive part is Al-rich. We note that this alloy segregation was observed for edge and mixed-type dislocations (no screw-type dislocations were analyzed in this study). This is not surprising given that, according to the elastic theory, the $\epsilon_{x x}$ profile for edge and mixed dislocations is the same - i.e. the dislocation is bounded by a tensile and compressive strain region.

To investigate whether the alloy segregation observed by TEM has any implications on the optical properties of the dislocations, we observed a set of dislocations by multi-microscopy. An example is presented in Figure 2. At first we can see that the dislocations observed in the AFM image correspond to a dark spot on the CL integrated intensity image. This result is in line with previous observations of dislocations by $\mathrm{CL},{ }^{6-9}$ and suggests that the dominant carrier recombination process at dislocations is non-radiative. In terms of CL emission energy we can see that the dislocations are bounded on both sides by a blue-shifted and a red-shifted region (as highlighted by the dislocation indicated by a square in Figure 2(c)). The shifts in emission energy are of the order of $-4 \pm 2 \mathrm{meV}$ and $3 \pm 2 \mathrm{meV}$ for the red-shifted and blue-shifted parts, respectively, and could be accounted for the most part by the variation of strain around the dislocation. In light of our TEM observations, these results indicate that the segregation of Ga atoms in the vicinity of the dislocation has no significant effect on the optical properties of the dislocation.

\subsection{InGaN epilayer}

Previous TEM observations of dislocations in InGaN have reported that In atoms segregate in the tensile part of dislocations. ${ }^{17,18}$ This result is very similar to what we have reported above for AlGaN, and can be explained
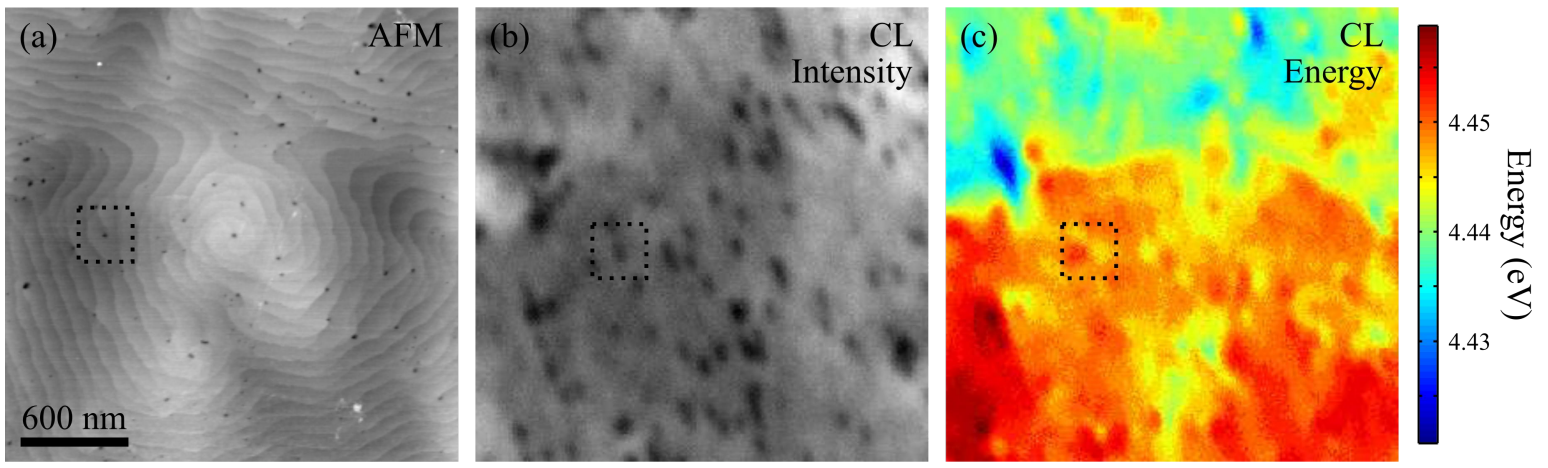

Figure 2. (a) AFM, (b) CL integrated intensity, and (c) CL peak emission energy images of the same region of the AlGaN epilayer. 

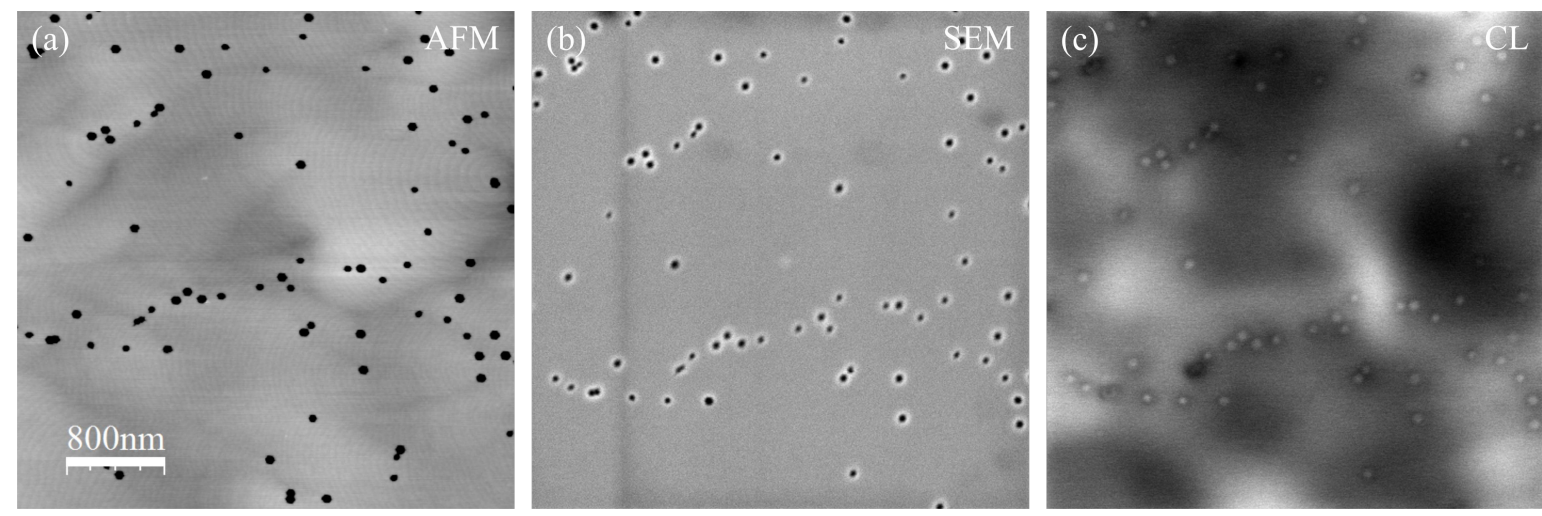

Figure 3. (a) AFM, (b) SEM, and (c) panchromatic CL images of the same region of the InGaN epilayer.

(a)

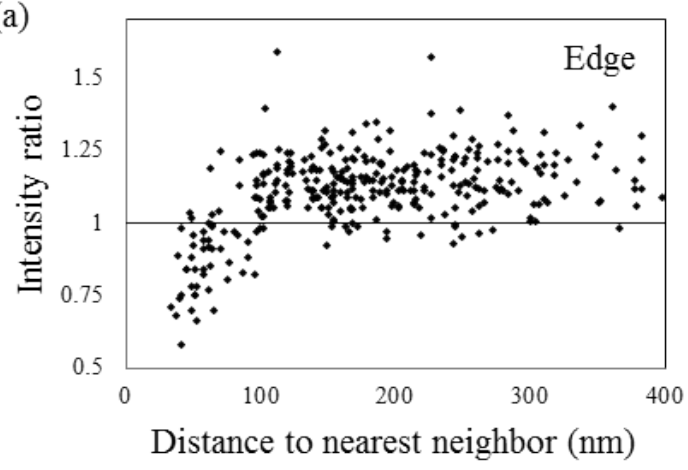

(b)

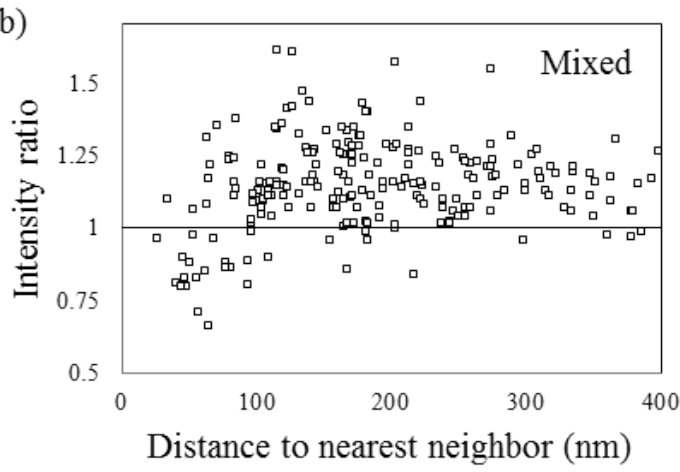

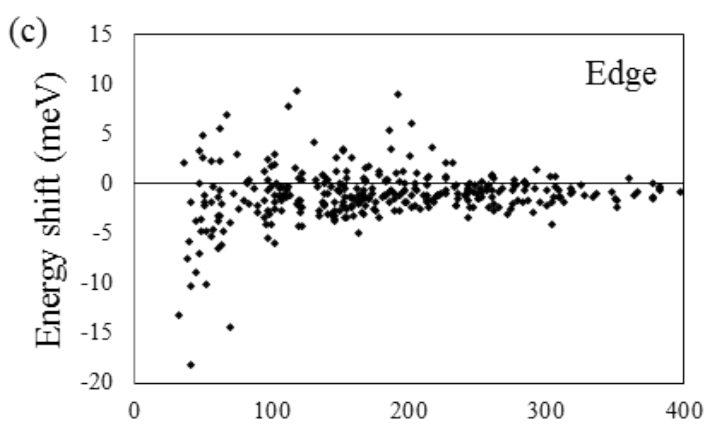

Distance to nearest neighbor $(\mathrm{nm})$

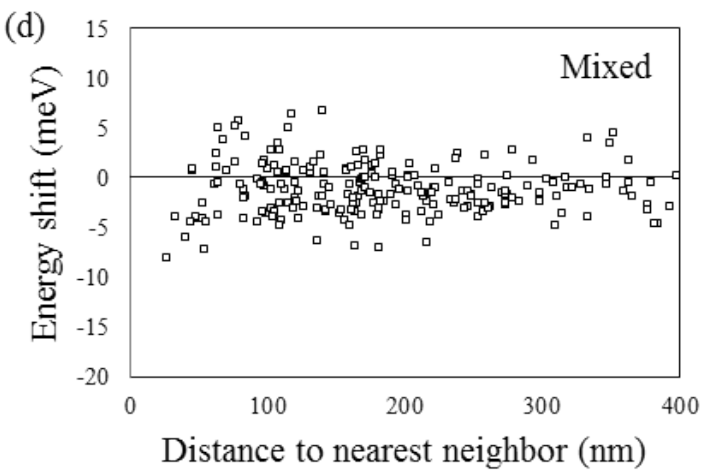

Figure 4. (a-b) Intensity ratio and (c-d) energy shift as a function of the distance to nearest neighbor for (a,c) edge and $(\mathrm{b}, \mathrm{d})$ mixed-type dislocations.

through similar energetic arguments because the In-N bond is longer than the Ga-N bond. In this section we will mostly focus on understanding the impact of In segregation on the optical properties.

An example of multi-microscopy approach carried out on the InGaN epilayer is given in Figure 3 . The dislocations can be readily identified as V-pits in the AFM and SEM images. From Figure 3(c), we can see that the dislocations correspond in the CL image to a bright spot surrounded by a dark halo. The presence of the bright spot indicates an enhanced light emission at the dislocation, which seems at first to challenge common views that dislocations act as non-radiative recombination centers. If mainly non-radiative recombination were to occur, one would expect to see a dark spot at the position of the dislocation, ${ }^{6-9}$ similar to what we noticed in the previous section for example. 
To understand the nature of the bright spot, we quantified the CL intensity and emission energy at the center of the V-pit (i.e. at the position of the dislocation) relative to the surrounding material (i.e. far away from the dislocation). The results are presented in Figure 4, for edge and mixed-type dislocations. We can at first see that all graphs show a similar dependence with distance to nearest neighbor, whereby the intensity ratio and energy shift increase with distance to nearest neighbor, then stabilize. The transition between these two regimes correspond to dislocations with spacing around 100-120 nm. Here we will focus on the dislocations with distance to nearest neighbor longer than $120 \mathrm{~nm}$ to avoid additional effects due to the interaction between neighboring dislocations.

For such "isolated" dislocations, the intensity ratio remains generally above 1 (Figure 4(a-b)), which supports our qualitative observation of the bright spot. Compared to that of the surrounding material, the emission intensity is increased in average by $15 \%$ and $17 \%$ at edge and mixed-type dislocations, respectively. Turning our attention to the energy shift (Figure 4(c-d)), we can see that the emission energy from the dislocation is very close to that of the surrounding material. We observe shifts ranging from $-5 \mathrm{meV}$ to $+9 \mathrm{meV}$ (with an average value of $-1 \mathrm{meV}$ ) for edge dislocations, and from $-7 \mathrm{meV}$ to $+7 \mathrm{meV}$ (with an average value of $-1 \mathrm{meV}$ ) for mixed dislocations.

We note that the bright spot is a very local feature, which involves low magnitude energy shifts (which can be positive or negative), and which properties are fairly independent with dislocation type. This seems to support the hypothesis that the luminescence is due to In-N chains and atomic condensates ${ }^{19-21}$ that very possibly formed as a result of the segregation of In at the dislocation. (Other possible explanations such as, strain relaxation or "bulk" change in alloy composition, can be ruled out as these would lead to much stronger and systematically negative energy shifts.) In-N chains and atomic condensates correspond to $\mathrm{N}$ atoms bonded to more than one In atom, and have been shown theoretically ${ }^{19,21}$ and experimentally ${ }^{20}$ to strongly localize holes. Moreover, Schulz et al. found that holes were localized over several excited states, up to $50 \mathrm{meV}$ from the top of the valence band. Hence the energy shift associated with In-N chains and atomic condensates would be expected to range between $-8 \mathrm{meV}$ and $+42 \mathrm{meV}$ for two In atoms sharing a common $\mathrm{N}$ atom, $-18 \mathrm{meV}$ and $+32 \mathrm{meV}$ (three In atoms), and $-29 \mathrm{meV}$ and $+21 \mathrm{meV}$ (four In atoms), which seems to agree with the values reported in Figure 4 .

\section{CONCLUSION}

In conclusion, we report that alloy fluctuations occur at dislocations in III-Nitride alloys (InGaN and AlGaN), such that the largest atoms (In for InGaN and Ga for AlGaN) segregate in the tensile part of dislocations with an edge component. In InGaN, In atom segregation leads to the enhanced formation of In-N chains and atomic condensates which localize carriers. This feature results in a bright spot in the CL images, showing that nonradiative recombination at dislocations is reduced. On the other hand, Ga segregation at dislocations in AlGaN does not significantly affect the intensity recorded by CL at the dislocation. The strong localization properties of In atoms seem to be at the origin of this discrepancy. Therefore, to limit the deleterious impact of dislocations in AlGaN-based devices, an interesting approach may be to dope AlGaN with In.

\section{ACKNOWLEDGMENTS}

This project is funded in part by the European Research Council under the European Community's Seventh Framework Programme (FP7/2007-2013)/ERC grant agreement No. 279361 (MACONS). The research leading to these results has received funding from the European Union Seventh Framework Programme under Grant Agreement 312483 - ESTEEM2 (Integrated Infrastructure InitiativeI3). S.R. acknowledges financial support from the ERC Starting Grant 307636 SCOPE. M.H. would like to acknowledge support from the Lindemann Trust Fellowship.

\section{REFERENCES}

[1] Humphreys, C., "Solid-State Lighting," MRS Bulletin 33, 459 (2008).

[2] Kneissl, M. and Rass, J., [III-Nitride Ultraviolet Emitters], Springer, Switzerland (2016). 
[3] Alhassan, A., Farrell, R., Saifaddin, B., Mughal, A., Wu, F., Denbaars, S., Nakamura, S., and Speck, J., "High luminous efficacy green light-emitting diodes with AlGaN cap layer," Optics Express 249, 17868 (2016).

[4] Narukawa, Y., Ichikawa, M., Sanga, D., Sano, M., and Mukai, T., "White light emitting diodes with superhigh luminous efficacy," Journal of Physics D: Applied Physics 43(35), 354002 (2010).

[5] Takano, T., Mino, T., Sakai, J., Noguchi, N., Tsubaki, K., and Hirayama, H., "Deep-ultraviolet lightemitting diodes with external quantum efficiency higher than 20efficiency," Applied Physics Express 10, 031002 (2017).

[6] Sugahara, T., Sato, H., Hao, M., Naoi, Y., Kurai, S., and Tottori, S., "Direct evidence that dislocations are non-radiative recombination centers in gan," Japanese Journal of Applied Physics 37(4), 398 (1998).

[7] Cherns, D., Henley, S., and Ponce, F., "Edge and screw dislocations as nonradiative centers in InGaN/GaN quantum well luminescence," Applied Physics Letters 78(18), 2691 (2001).

[8] Rosner, S., Carr, E., Ludowise, M., Girolami, G., and Erikson, H., "Correlation of cathodoluminescence inhomogeneity with microstructural defects in epitaxial gan grown by metalorganic chemical-vapor deposition," Applied Physics Letters 70(4), 420 (1997).

[9] Liu, W., Carlin, J.-F., Grandjean, N., Deveaud, B., and Jacopin, G., "Exciton dynamics at a single dislocation in GaN probed by picosecond time-resolved cathodoluminescence," Applied Physics Letters 109, 042101 (2016).

[10] Davies, M., Dawson, P., Massabuau, F., Oehler, F., Oliver, R., Kappers, M., Badcock, T., and Humphreys, C., "The effects of varying threading dislocation density on the optical properties of InGaN/GaN quantum wells," Physica Status Solidi (C) 11(3-4), 750 (2014).

[11] Kneissl, M., Kolbe, T., Chua, C., Kueller, V., Lobo, N., Stellmach, J., Knauer, A., Rodriguez, H., Einfeldt, S., Yang, Z., Johnson, N. M., and Weyers, M., "Advances in group III-nitride-based deep UV light-emitting diode technology," Semiconductor Science and Technology 26(1), 014036 (2011).

[12] Sridhara Rao, D., Kappers, M., McAleese, C., Zhy, T., Zhu, D., and Humphreys, C., "Microstructural Study of AlGaN/AlN Buffer with 3D GaN Interlayer," XV International Workshop on the Physics of Semiconductor Devices 2009 79, 264-267 (2009).

[13] Massabuau, F., Chen, P., Horton, M., Rhode, S., Ren, C., O'Hanlon, T., Kovács, A., Kappers, M., Humphreys, C., Dunin-Borkowski, R., and Oliver, R., "Carrier localization in the vicinity of dislocations in InGaN," Journal of Applied Physics 121, 013104 (2017).

[14] Ernst Ruska-Centre for Microscopy and Spectroscopy with Electrons (ER-C), "FEI Titan G2 80-200 CREWLEY," Journal of large-scale research facilities 2, A43 (2016).

[15] Massabuau, F., Trinh-Xuan, L., Lodie, D., Thrush, E., Zhu, D., Oehler, F., Zhu, T., Kappers, M., Humphreys, C., and Oliver, R., "Correlations between the morphology and emission properties of trench defects in InGaN/GaN quantum wells," Journal of Applied Physics 113(7), 073505 (2013).

[16] Oliver, R., Kappers, M., Sumner, J., Datta, R., and Humphreys, C., "Highlighting threading dislocations in MOVPE-grown GaN using an in situ treatment with SiH4 and NH3," Journal of Crystal Growth 289(2), 506 (2006).

[17] Horton, M., Rhode, S., Sahonta, S.-L., Kappers, M., Haigh, S., Pennycook, T., Humphreys, C., Dusane, R., and Moram, M., "Segregation of In to Dislocations in InGaN," Nano letters 15, 923 (2015).

[18] Rhode, S., Horton, M., Sahonta, S., Kappers, M., Haigh, S., Pennycook, T., McAleese, C., Humphreys, C., Dusane, R., and Moram, M., "Dislocation core structures in (0001) InGaN," Journal of Applied Physics 119(10), 105301 (2016).

[19] Liu, Q., Lu, J., Gao, Z., Lai, L., Qin, R., Li, H., Zhou, J., and Li, G., "Electron localization and emission mechanism in wurtzite (Al, In, Ga)N alloys," Physica Status Solidi (B) 247(1), 109 (2010).

[20] Chichibu, S., Uedono, A., Onuma, T., Haskell, B., Chakraborty, A., Koyama, T., Fini, P., Keller, S., Denbaars, S., Speck, J., Mishra, U., Nakamura, S., Yamaguchi, S., Kamiyama, S., Amano, H., Akasaki, I., Han, J., and Sota, T., "Origin of defect-insensitive emission probability in in-containing (al,in,ga)n alloy semiconductors," Nature materials 5(10), 810- (2006).

[21] Schulz, S., Marquardt, O., Coughlan, C., Caro, M., Brandt, O., and O'Reilly, E., "Atomistic description of wave function localization effects in $\mathrm{In}_{-}\{\mathrm{x}\} \mathrm{Ga}_{-}\{1-\mathrm{x}\} \mathrm{N}$ alloys and quantum wells," Proceedings of SPIE $\mathbf{9 3 5 7}$, 93570C (2015). 